\title{
THE G-FUNCTIONS AS UNSYMMETRICAL FOURIER KERNELS. III
}

\author{
ROOP NARAIN
}

1. The functions $k(x)$ and $h(x)$ are said to form a pair of Fourier kernels if the reciprocal equations

$$
\begin{aligned}
& g(x)=\int_{0}^{\infty} k(x y) f(y) d y, \\
& f(x)=\int_{0}^{\infty} h(x y) g(y) d y,
\end{aligned}
$$

are simultaneously satisfied. The kernels are said to be symmetrical if $k(x)=h(x)$ and unsymmetrical if $k(x) \neq h(x)$. In an earlier paper $[1$, p. 953] I found a pair of unsymmetrical Fourier kernels expressed in terms of Meijer's $G$-function as

$$
\begin{aligned}
k(x) & =K(x) \equiv 2 \gamma x^{\gamma-1 / 2} G_{p+q, m+n}^{m, p}\left(x^{2 \gamma} \mid \begin{array}{l}
a_{1}, \cdots, a_{p}, b_{1}, \cdots, b_{q} \\
c_{1}, \cdots, c_{m}, d_{1}, \cdots, d_{n}
\end{array}\right), \\
h(x) & =H(x) \\
& \equiv 2 \gamma x^{\gamma-1 / 2} G_{p+q, m+n}^{n, q}\left(x^{2 \gamma} \mid \begin{array}{l}
-b_{1}, \cdots,-b_{q},-a_{1}, \cdots,-a_{p} \\
-d_{1}, \cdots,-d_{n},-c_{1}, \cdots,-c_{m}
\end{array}\right)
\end{aligned}
$$

where $\gamma>0, n-p=m-q>0$ and

$$
\sum_{j=1}^{p} a_{j}+\sum_{j=1}^{q} b_{j}=\sum_{j=1}^{m} c_{j}+\sum_{j=1}^{n} d_{j} .
$$

The importance of these functions is due to their very general yet simple form from which many known as well as new kernels can be deduced as special cases $[1$, p. 954, §3]. I also established in another paper [2, p. 21] the reciprocities (1.1), (1.1') with the functions $K(x)$ and $H(x)$ by using the ordinary convergence methods. The aim of the present paper is to establish the reciprocities $(1.1),\left(1.1^{\prime}\right)$ by employing the convergence in the mean square method. As is well known in the theory of Fourier integrals, the mean convergence theory is both easier and more general than the other. The result is given in the form of a theorem, the proof of which is based upon an extended form of a Watson's theorem [3, p. 221, Theorem 129 extended in the sense of $\$ 8.9$ on p. 226] which may be stated as in the next section.

Received by the editors March 1, 1962. 
2. Let $\kappa(s)$ and $\eta(s)$ be the Mellin transforms of $k(x)$ and $h(x)$ respectively, then the formal necessary condition $[3$, p. 214, Equation (8.3.5)] that $k(x)$ and $h(x)$ be unsymmetrical Fourier kernels is that

$$
\kappa(s) \eta(1-s)=1 .
$$

However, we need only assume the existence of the functions $\kappa(s)$ and $\eta(s)$ on the line $\sigma=\frac{1}{2}$ where $s=\sigma+i$ so that (2.1) becomes

$$
\kappa\left(\frac{1}{2}+i t\right) \eta\left(\frac{1}{2}-i t\right)=1 .
$$

We also assume that $\kappa\left(\frac{1}{2}+i t\right)$ and $\eta\left(\frac{1}{2}+i t\right)$ are both bounded as $|t| \rightarrow \infty$.

Let now $k_{1}(x)$ and $h_{1}(x)$ be so defined that $k_{1}(x) / x$ and $h_{1}(x) / x$ are the Mellin transforms of $\kappa\left(\frac{1}{2}+i t\right) /\left(\frac{1}{2}-i t\right)$ and $\eta\left(\frac{1}{2}+i t\right) /\left(\frac{1}{2}-i t\right)$ respectively, i.e.

$$
\begin{aligned}
& \frac{k_{1}(x)}{x}=\frac{1}{2 \pi} \lim _{T \rightarrow \infty} \int_{-T}^{T} \frac{\kappa\left(\frac{1}{2}+i t\right)}{\frac{1}{2}-i t} x^{-(1 / 2)-i t} d t, \\
& \frac{h_{1}(x)}{x}=\frac{1}{2 \pi} \lim _{T \rightarrow \infty} \int_{-T}^{T} \frac{\eta\left(\frac{1}{2}+i t\right)}{\frac{1}{2}-i t} x^{-(1 / 2)-i t} d t,
\end{aligned}
$$

the integrals being convergent in the mean square sense since on account of the boundedness of $\kappa\left(\frac{1}{2}+i t\right)$ and $\eta\left(\frac{1}{2}+i t\right), \kappa\left(\frac{1}{2}+i t\right) /\left(\frac{1}{2}-i t\right)$ and $\eta\left(\frac{1}{2}+i t\right) /\left(\frac{1}{2}-i t\right)$ belong to $L^{2}(-\infty, \infty)$. Also it follows that $k_{1}(x) / x$ and $h_{1}(x) / x$ belong to $L^{2}(0, \infty)$.

The analogue of Theorem 129 of Titchmarsh [3, p. 221] appropriate for the unsymmetrical case $[3$, p. 226, \$8.9] is as follows.

Theorem A. Let $\kappa\left(\frac{1}{2}+i t\right)$ and $\eta\left(\frac{1}{2}+i t\right)$ be bounded functions of $t$ satisfying (2.2). Let $k_{1}(x)$ and $h_{1}(x)$ be defined by (2.3) and (2.3') and $f(x)$ be any function belonging to $L^{2}(0, \infty)$. Then the formulae

$$
\begin{aligned}
& g_{k}(x)=\frac{d}{d x} \int_{0}^{\infty} k_{1}(x u) f(u) \frac{d u}{u}, \\
& g_{h}(x)=\frac{d}{d x} \int_{0}^{\infty} h_{1}(x u) f(u) \frac{d u}{u},
\end{aligned}
$$

define almost everywhere functions $g_{k}(x)$ and $g_{h}(x)$ respectively both belonging to $L^{2}(0, \infty)$. Also the reciprocal formulae

$$
f(x)=\frac{d}{d x} \int_{0}^{\infty} h_{1}(x u) g_{k}(u) \frac{d u}{u},
$$




$$
f(x)=\frac{d}{d x} \int_{0}^{\infty} k_{1}(x u) g_{h}(u) \frac{d u}{u},
$$

hold almost everywhere. And further,

$$
\int_{0}^{\infty}[f(x)]^{2} d x=\int_{0}^{\infty} g_{k}(x) g_{h}(x) d x .
$$

The proof of this (as is indicated in $[3, \S 8.9]$ also) is almost similar to that of Theorem 129 of Titchmarsh, cited above.

3. Using the definition [4, p. 207] of $G$-function, we may write after an obvious transformation of the variable of integration

$$
\begin{aligned}
& K(x)=\frac{1}{2 \pi i} \int_{L} \mathcal{K}(s) x^{-s} d s, \\
& H(x)=\frac{1}{2 \pi i} \int_{L} \mathcal{H}(s) x^{-s} d s,
\end{aligned}
$$

where

$$
\begin{aligned}
& \mathcal{K}(s)= \frac{\prod_{j=1}^{m} \Gamma\left(\frac{1}{2}+c_{j}-\frac{1}{4 \gamma}+\frac{s}{2 \gamma}\right) \prod_{j=1}^{p} \Gamma\left(\frac{1}{2}-a_{j}+\frac{1}{4 \gamma}-\frac{s}{2 \gamma}\right)}{\prod_{j=1}^{n} \Gamma\left(\frac{1}{2}-d_{j}+\frac{1}{4 \gamma}-\frac{s}{2 \gamma}\right) \prod_{j=1}^{q} \Gamma\left(\frac{1}{2}+b_{j}-\frac{1}{4 \gamma}+\frac{s}{2 \gamma}\right)}, \\
& \mathcal{C}(s)=\frac{\prod_{j=1}^{n} \Gamma\left(\frac{1}{2}-d_{j}-\frac{1}{4 \gamma}+\frac{s}{2 \gamma}\right) \prod_{j=1}^{q} \Gamma\left(\frac{1}{2}+b_{j}+\frac{1}{4 \gamma}-\frac{s}{2 \gamma}\right)}{\prod_{j=1}^{m} \Gamma\left(\frac{1}{2}+c_{j}+\frac{1}{4 \gamma}-\frac{s}{2 \gamma}\right) \prod_{j=1}^{p} \Gamma\left(\frac{1}{2}-a_{j}-\frac{1}{4 \gamma}+\frac{s}{2 \gamma}\right)}
\end{aligned}
$$

and the contour $L$ is the line from $\sigma-i \infty$ to $\sigma+i \infty$. It is assumed that $\gamma>0$ and all poles of the integrands in (3.1) and (3.1') are simple and such that the $c_{j}$-poles of (3.2) and $d_{j}$-poles of $\left(3.2^{\prime}\right)$ lie to the left of $L$ while $a_{j}$-poles of (3.2) and $b_{j}$-poles of (3.2') lie to the right of $L$. Evidently $\mathscr{K}(s)$ and $\mathfrak{C}(s)$ are the Mellin transforms of $K(x)$ and $H(x)$ respectively.

For large $s$, the asymptotic expansion $[4$, p. 47] of the Gamma function is given by

$$
\log \Gamma(s+a)=\left(s+a-\frac{1}{2}\right) \log s-s+\frac{1}{2} \log (2 \pi)+O\left(\frac{1}{s}\right)
$$

where $|\arg s|<\pi$. To obtain the behaviour of $\mathscr{K}(s)$ and $\mathfrak{F C}(s)$ for 
large $|t|$, we replace Gamma functions involving $-s / 2 \gamma$ by those containing $+s / 2 \gamma$ with the help of the relation

$$
\Gamma(z) \Gamma(1-z)=\pi \csc \pi z .
$$

Then using (3.3) and the conditions (i) and (ii) of the Theorem 1 below we get along the line $L$,

$$
\begin{aligned}
\mathcal{K}(s) x^{-s}= & |t|^{\mu(\sigma-1 / 2)} \exp \{i t(\mu \log t-\log x+B)\} \\
& \cdot\left\{D+O\left(\frac{1}{|t|}\right)\right\}
\end{aligned}
$$

and

$$
\begin{aligned}
\mathscr{H C}(s) x^{-s}= & |t|^{\mu(\sigma-1 / 2)} \exp \left\{i t\left(\mu \log t-\log x+B^{\prime}\right)\right\} \\
& \cdot\left\{D^{\prime}+O\left(\frac{1}{|t|}\right)\right\}
\end{aligned}
$$

for large $|t|$, where $\mu=(n-p) / \gamma, B$ and $B^{\prime}$ are constants and $D$ and $D^{\prime}$ are also constants each having one value for large positive $t$ and another value for large negative $t$.

From (3.4) and $\left(3.4^{\prime}\right)$ it follows that the integrals (3.1) and (3.1') are uniformly convergent for all $x$ when $\sigma<\frac{1}{2}$. Thus if $\sigma<\frac{1}{2}$, the integration with respect to $x$ under the integral sign in (3.1) and (3.1') is justified. Let us denote

$$
\begin{aligned}
& K_{1}(x)=\int_{0}^{x} K(x) d x, \\
& H_{1}(x)=\int_{0}^{x} H(x) d x .
\end{aligned}
$$

Then

$$
\begin{aligned}
& K_{1}(x)=\frac{1}{2 \pi i} \int_{L} \frac{\mathscr{K}(s)}{1-s} x^{1-s} d s \\
& H_{1}(x)=\frac{1}{2 \pi i} \int_{L} \frac{\mathscr{H}(s)}{1-s} x^{1-s} d s
\end{aligned}
$$

whenever $\sigma<\frac{1}{2}$.

We, however, require the above relations to hold when $\sigma=\frac{1}{2}$ because we have to consider $\mathscr{K}(s)$ and $\mathfrak{C}(s)$ along the line $\sigma=\frac{1}{2}$. It is easy to show that the integrals on the right of (3.6) and (3.6') converge to $K_{1}(x)$ and $H_{1}(x)$ respectively when $\sigma=\frac{1}{2}$. 
On multiplying (3.4) by $1 / t$, we see that the integral in (3.6) converges when $\sigma=\frac{1}{2}$ and, since $\mu>0$, that we may close the contour by a large semicircle on the left. With the conditions (iii) and (v) of the theorem below, the only singularities of the integrand within this closed contour are the simple poles of

$$
\Gamma\left(\frac{1}{2}+c_{j}-\frac{1}{4 \gamma}+\frac{s}{2 \gamma}\right), \quad j=1, \cdots, m .
$$

Computation of the integral in (3.6) by residues gives us easily the individual powers of $K(x)$ each integrated from 0 to $x$. Since $K(x)$ is an entire function such term by term integration is justified and the final result is $K_{1}(x)$ as defined in (3.5). Hence with the conditions of Theorem 1, (3.5) and (3.6) are true and similarly (3.5') and (3.6') are true when $\sigma=\frac{1}{2}$.

On the line $\sigma=\frac{1}{2}$, it is clear from (3.4) that $\mathcal{K}(s) x^{-8}$ is bounded for all values of $t$. Hence on $\sigma=\frac{1}{2}, \Re(s) x^{-s} /(1-s)$ belongs to

$$
L^{2}\left(\frac{1}{2}-i \infty, \frac{1}{2}+i \infty\right)
$$

and so the integral in (3.6) converges in mean square. But as shown above the integral in (3.6) also converges in the ordinary sense to $K_{1}(x)$ of (3.5). Hence if the integral is evaluated by mean square methods its value will be $K_{1}(x)$ of (3.5) except, perhaps, in a set of measure zero. Similarly $\mathfrak{H C}(s) x^{-s} /(1-s)$ belongs to $L^{2}\left(\frac{1}{2}-i \infty, \frac{1}{2}+i \infty\right)$ and the integral in $\left(3.6^{\prime}\right)$ converges in mean square to $H_{1}(x)$ of $\left(3.5^{\prime}\right)$.

4. THEOREM 1. If

(i) $\gamma>0, n-p=m-q>0$

(ii) $\sum_{j=1}^{p} a_{j}+\sum_{j=1}^{q} b_{j}=\sum_{j=1}^{m} c_{j}+\sum_{j=1}^{n} d_{j}$,

(iii) $\operatorname{Rl}\left(\frac{1}{2}-a_{j}\right)>0, j=1, \cdots, p$,

(iv) $\operatorname{Rl}\left(\frac{1}{2}+b_{j}\right)>0, j=1, \cdots, q$,

(v) $\mathrm{Rl}\left(\frac{1}{2}+c_{j}\right)>0, j=1, \cdots, m$,

(vi) $\operatorname{Rl}\left(\frac{1}{2}-d_{j}\right)>0, j=1, \cdots, n$,

(vii) $f(x)$ belongs to $L^{2}(0, \infty)$,

then the formulae

$$
\begin{aligned}
& \frac{d}{d x} \int_{0}^{\infty} K_{1}(x u) f(u) \frac{d u}{u}=g_{K}(x), \\
& \frac{d}{d x} \int_{0}^{\infty} H_{1}(x u) f(u) \frac{d u}{u}=g_{H}(x),
\end{aligned}
$$


define almost everywhere functions $g_{K}(x)$ and $g_{H}(x)$ respectively both belonging to $L^{2}(0, \infty)$. Also the reciprocal formulae

$$
\begin{aligned}
& \frac{d}{d x} \int_{0}^{\infty} H_{1}(x u) g_{K}(u) \frac{d u}{u}=f(x), \\
& \frac{d}{d x} \int_{0}^{\infty} K_{1}(x u) g_{H}(u) \frac{d u}{u}=f(x),
\end{aligned}
$$

hold almost everywhere. And further

$$
\int_{0}^{\infty}[f(x)]^{2} d x=\int_{0}^{\infty} g_{H}(x) g_{K}(x) d x .
$$

In (3.6) and (3.6') which define $K_{1}(x)$ and $H_{1}(x)$ in a sense similar to that in (2.3) and $\left(2.3^{\prime}\right)$, we take $\sigma=\frac{1}{2}$ so that the contour $L$ is the line from $\frac{1}{2}-i \infty$ to $\frac{1}{2}+i \infty$. The inequalities (iii), (iv), (v), and (vi) then ensure that the poles of $\mathscr{K}(s)$ and $\mathfrak{H C}(s)$ lie on such sides of $L$ as is required for the definition of $K(x)$ and $H(x)$. We will now establish the truth of the requirements of Theorem A of $\$ 2$.

The first requirement is that $\mathscr{K}\left(\frac{1}{2}+i t\right)$ and $\mathcal{H}\left(\frac{1}{2}+i t\right)$ are bounded and that (2.2) is satisfied. This is clearly true from (3.2), (3.2'), (3.4) and $\left(3.4^{\prime}\right)$. The second requirement is that $K_{1}(x)$ and $H_{1}(x)$ are related with $\mathcal{K}(s)$ and $\mathcal{H}(s)$, the Mellin transforms of $K(x)$ and $H(x)$ respectively, according to (2.3) and $\left(2.3^{\prime}\right)$. This is explained at the end of $\$ 3$ above. The third requirement is that $f(x)$ belongs to $L^{2}(0, \infty)$ which is covered by the hypothesis (vii) of the theorem.

Since all the conditions of the Theorem A are satisfied, its conclusions follow and in our case these conclusions are (4.1), (4.1'), (4.2), $\left(4.2^{\prime}\right)$ and (4.3).

5. Taking $p=q, m=n$ and

$$
\begin{aligned}
a_{j}+b_{j}=0, & j=1, \cdots, p, \\
c_{j}+d_{j}=0, & j=1, \cdots, m,
\end{aligned}
$$

we notice that

$$
K(x)=H(x)=2 \gamma x^{\gamma-1 / 2} G_{2 p, 2 m}^{m, p}\left(x^{2 \gamma} \mid \begin{array}{l}
a_{1}, \cdots, a_{p},-a_{1}, \cdots,-a_{p} \\
c_{1}, \cdots, c_{m},-c_{1}, \cdots,-c_{m}
\end{array}\right),
$$

which is a symmetrical Fourier kernel obtained formally by me in an earlier paper [5, p. 298]. Corresponding to this symmetrical kernel $K(x)$ of (5.1), the Theorem 1 takes the form 
THEOREM 2. If

(i) $\gamma>0, m-p>0$,

(ii) $\operatorname{Rl}\left(\frac{1}{2}-a_{j}\right)>0, j=1, \cdots, p$,

(iii) $\operatorname{Rl}\left(\frac{1}{2}+c_{j}\right)>0, j=1, \cdots, m$,

(iv) $f(x)$ belongs to $L^{2}(0, \infty)$,

then the formula

$$
\frac{d}{d x} \int_{0}^{\infty} K_{1}(x u) f(u) \frac{d u}{u}=g(x)
$$

defines almost everywhere the function $g(x)$ belonging to $L^{2}(0, \infty)$. Also the reciprocal formula

$$
\frac{d}{d x} \int_{0}^{\infty} K_{1}(x u) g(u) \frac{d u}{u}=f(x)
$$

holds almost everywhere and further

$$
\int_{0}^{\infty}[f(x)]^{2} d x=\int_{0}^{\infty}[g(x)]^{2} d x .
$$

This theorem is due to Fox [6, p. 399] and it is this theorem which inspired me to write this paper.

\section{REFERENCES}

1. R. Narain, The G-functions as unsymmetrical Fourier kernels. I, Proc. Amer. Math. Soc. 13 (1962), 950-959.

2. - The G-functions as unsymmetrical Fourier kernels. II, Proc. Amer. Math. Soc. 14 (1963), 18-28.

3. E. C. Titchmarsh, Theory of Fourier integrals, Oxford, 1937.

4. Bateman Manuscript Project, Higher transcendental functions, Vol. 1, McGraw-Hill, New York, 1953.

5. R. Narain, A Fourier kernel, Math. Z. 70 (1959), 297-299.

6. C. Fox, The $G$ and $H$ functions as symmetrical Fourier kernels, Trans. Amer. Math. Soc. 98 (1961), 395-429.

WASHINGTON UNIVERSITY 